\title{
Anatomic Stage IV Breast Cancer AJCC v8
}

National Cancer Institute

\section{Source}

National Cancer Institute. Anatomic Stage IV Breast Cancer A/CC v8. NCI Thesaurus. Code C139545.

Stage IV includes: Any T, Any N, M1. M1: Distant metastases detected by clinical and radiographic means and/or histologically proven metastases larger than $0.2 \mathrm{~mm}$. (AJCC 8th ed.) 